PS10.02.11 STRUCTURE OF NANOCRYSTALLINE SPINEL FERRITE PRODUCED BY HIGH-ENERGY BALLMULLING METHOD. V. Sepelak', A. Yu. Rogachev2, U. Steinike 3 , D.-Chr. Uecker 33 , S. Wissmann 4 and K. D. Becker', 1 Institute of Geotechnics of the Slovak Academy of Sciences, 04353 Kosice, Slovak Republic, 2Institute of Solid State Chemistry of the Russian Academy of Sciences, K 630091 Novosibirsk, Russia, 3Institute of Applied Chemistry e.V., 12484 Berlin, Germany, 4 Institute of Physical and Theoretical Chemistry, Technical University of Braunschweig, 38106 Braunschweig, Germany

First mechanochemical synthesis (synthesis induced by a highenergy ball-milling process) of zinc ferrite from zinc oxide and iron oxide powders at room temperature is presented. Structure of the formed zine ferrite with a large amount of strain and defects introduced during the mechanical treatment is characterized by means of $X$-ray powder diffraction, scanning electron microscopy, and Mössbauer spectroscopy. The ball-milling of the $\mathrm{ZnO} / \mathrm{Fe}_{2} \mathrm{O}_{3}$ mixture in a planetary mill results in the formation of zinc ferrite with non-equilibrium distribution of cations between tetrahedral and octahedral positions $[1,2]$. Subsequent thermal treatment of the disordered zinc ferrite leads to its crystallization at temperatures, which are by about $300 \mathrm{~K}$ lower than those at which the crystalline zinc ferrite is formed by a conventional thermal method commonly involving solid-state reactions.

[1] Sepelak, V., Steinike, U.. Thacova, K., Uecker, D.-Chr., Richter-Mendau, J., Jancke, K., Buchal, A., Rykov, A. I., Rogachev, A.Yu.: Z.Kristallogr:Suppl. 8 (1994) 673 .

[2] Sepelak, V., Tkacova, K., Steinike, U., Boldyrev, V.V.: European Powder Diffraction Conference EPDIC-4, Chester 1995, Mat.Sci.Forum 1996, in press.

PS10.02.12 MODULATIONS OF INTERMETALLIC B8TYPE PHASES. Sven Lidin, Margareta Elding \& Lars Stenberg. Inorganic chemistry, Amhenius Laboratory, Stockholm University, 10691 Stockholm

Intermetallic B8 type phases exhibit a full spectrum of modes of ordering from short period, commensurate, superstructures to long period or incommensurate modulations and short range order as indicated by highly structured diffuse scattering. We will present results on modulated structures from the systems $\mathrm{Cu}-\mathrm{Sn}, \mathrm{Co}-\mathrm{Sn}$, $\mathrm{Cu}-\mathrm{In}, \mathrm{Ni}-\mathrm{Bi}$ and $\mathrm{Mn}-\mathrm{Sn}$ derived by electron diffraction and high resolution electron microscopy, single crystal X-ray diffraction and $\mathrm{X}$-ray powder diffraction.

\section{Materials III}

\section{Strain and Stress Measurements}

MS10.03.01 PROFILING STRAIN IN THIN BURTED LAYERS BY CONVERGENT BEAM ELECTRON DIFFRACTION TECHNIQUES. D. Chems Physics Department, University of Bristol, Bristol, BS8 1TL UK

Convergent beam electron diffraction (CBED) provides a powerful method of recording crystal rocking curves. In contrast to X-ray rocking curves, electron rocking curves are not restricted to low order reflections and have spatial resolutions down to about $1 \mathrm{~nm}$. The method has now been used to profile strain and composition in a range of semiconductor multilayer structures in both plan-view and crosssectional geometries.

Electron rocking curves have been most usefully recorded using the large angle CBED (LACBED) method which shows spatial variations directly. LACBED also provides a means of filtering out most of the phonon- and some of the plasmongenerated inelastic background, giving useful rocking curve detail up to a tilt of more than $10^{\circ}$ from the Bragg orientation. Studies of Si/SiGe multilayers in a plan-view geometry have shown that layer strains may be determined to $20 \%$ accuracy (1). Corresponding studies on cross-sectional samples (2) suggest a similar sensitivity; in this case strainrelaxation must be taken into account. Work on single buried layers in InP/ InGaAs in plan-view has shown that, using low order reflections, strains may be analysed for layers down to 1 monolayer in thickness (3). Recently we have extended these studies to high order reflections which are more sensitive to layer srains. Results obtained for thin Ge layers in Si indicate that strain can thereby be profiled across Ge islands where 3-D growth has occurred.

(1) D. Cherns, R. Tounitia, A. R. Preston, C. J. Rossouw and D. Houghton, Phil. Mag. A64 (1991) 597.

(2) X-F. Duan, D. Cherns and J. W. Steeds, Phil. Mag. A70 (1994) 1091. (3) N. Grigorieff. D. Cherns, M. J. Yates, M. Hockly, S. D. Perrin and M. R. Aylett, Phil. Mag. A68 (1993) 121.

MS10.03.02 X-RAY STRAIN MEASUREMENTS ON SEMICONDUCTORS. Paul F Fewster, Philips Research Laboratories, Cross Oak Lane, Redhill, U.K.

A combination of reciprocal space mapping and absolute lattice parameter determination can yield highly accurate and unambiquous strain measurements. Strain measurement are used extensively in $\mathrm{x}$ ray diffraction for determining composition phase extent, layer relaxation and the presence of defects after ion implantation. The method most commonly used is the "rocking curve" method that assumes that the substrate has a known lattice parameter. Simulation of the "rocking curve" is necessary in most circumstances to separate strain from diffraction effects[1].

Layer tilts can complicate this measurement considerably and a general tilt will lead to errors unless this is taken into consideration. However reciprocal space mapping in three-dimensions can resolve the complication of tilts[2]. The uncertainties in the lattice parameter of the substrate are resolved by combining this reciprocal space mapping with a very high precision absolute lattice parameter method[3]. Examples will be given to show how the strain in simple, complex and inhomogeneous semiconductors can be determined.

[1] Fewster \& Curling (1987) J Appl. Phys. 624154

[2] Fewster \& Andrew (1995) J Phys. D 28 A 154

[3] Fewster \& Andrew (1995) J Appl. Cryst. 28451

MS10.03.03 STRAIN DETERMUNATION IN SEMICONDUCTORS BY CONVERGENT BEAM ELECTRON DIFFRACTION. A. Armigliato, R.Balboni, A.Benedetti, S.Frabboni* and J.Vanhellemont ${ }^{+}$, CNR-Istituto LAMEL, via P.Gobetti 101, I40129 Bologna. Italy, "Dipartimento di Fisica, Università di Modena, via Campi 213/A, I-41100 Modena, Italy, +IMEC, Kapeldreef 75, B-3001 Leuven, Belgium

The deternination of strain in nanoscale structures of semiconductors requires the availability of techniques with a high spatial resolution. Convergent beam electron diffraction, which is performed in a transmission electron microscope (TEM/CBED), is a point-topoint technique, with a resolution of the order of ten nm, which allows the strain tensor to be deduced from the shift of HOLZ lines in the central spot of the pattern. In its large angle version (LACBED) this technique has the advantage of combining both real space and reciprocal space information, with a spatial resolution linited by the probe size: in this case strain information can be obtained from a single pattern in different points of the imaged area through the shift and splitting of the ZOLZ Bragg contours. However, when applied to cross-sectioned specimens, the strain measured by CBED or LACBED is affected by the relaxation, which occurs in a direction parallel to the thinning direction. Methods of quantitative determination of relaxation in both uniform and graded $\mathrm{Si}_{1-x} \mathrm{Ge}_{x} / \mathrm{Si}$ heterostructures will be discussed; the bulk tetragonal distortion values thus obtained from (LA)CBED are in good agreement with the 
ones deduced from double crystal $\mathrm{X}$-ray diffraction. Integrated circuits with submicron features are a promising field of application of this technique; the determination of strain profiles in local isolation structures in silicon and their agreement with the corresponding simulated ones will be reported.

MS10.03.04 PRECISION, ACCURACYAND RESOLUTION OF STRAIN MEASUREMENT WITH ELECTRON MICRODIFTRACTION. J.M. Zuo, Dept. of Physics and Astronomy, Arizona State University, Tempe, AZ 85287

Electron microdiffraction formed with a convergent beam is capable of probing crystalline strain filed associated with mismatching interfaces and grain boundaries in nanometer resolutions. The diffraction pattern formed with diskes containes both low order structure factor and lattice parameter information. However, application of electron microdiffraction is complicated by the dynamic effects or multiple scattering in electron diffraction. It has been shown that under weak scattering conditions in a off zone axis orientation the position of high order zone lines (HOLZ) (similar to the Kossel lines in $\mathrm{x}$-ray) can be approximated kinematically. In cases where the choice of diffraction condition is limited by the nature of strain field, for example, edge on orientation in the case of interface, effects of dynamic scattering in the HOLZ line positions need to be estimated and taken into considerations in the simulation. Examples in and off zone axis cases will be given together with the practical applications in semiconductor interfaces. A simple practical procedures in diamond and zincblende structures will also be described. The recent developement of 25 micron pixel imaging plates for electrons makes it possible to record whole electron diffraction with sufficient resolution digitally. The pixel registry of imaging plates plus the intensity data make it possible to analyse the whole pattern geometry with sufficient accuracy. Its benefit for the strain measurement will be discussed together with other possibilities with this new detector.

MS10.03.05 MEASUREMENT OF LATTCE STRAIN IN METALS BY QUANTITATIVE CONVERGENT BEAM ELECTRON DIFFRACTION. J. Mayer, C. Deininger, S. Streiffer*, A. Weickenmeier, Max-Planck-Institut für Metallforschung, Seestr. 92, 70174 Stuttgart, Germany, "now at: Dept. of Materials Science and Engineering, North Carolina State University, Raleigh, NC 27695-7907

Lattice strain in metals or alloys is an important factor controlling the performance and failure mechanisms in compound systems. In combination with ceramics or semiconductors, the met$\mathrm{al}$ is always the elastically and plastically softer material. Therefore most of the strain is localised within the metal. In such systems, temperature changes lead to lattice strain caused by the difference in thermal expansion coefficients. Convergent Beam Electron Diffraction (CBED) makes it possible to measure lattice strain with high spatial resolution. Strain can e.g. be measured as a function of distance from an interface. We have studied Al thin films on $\mathrm{SiC}$ and Si substrates. In particular the latter one serves as a good model system for interconnects in semiconductor devices. The samples were studied in cross-section and plan-view geometry. The CBED patterns were acquired on an energy-filtering Zeiss EM 912 Omega transmission electron microscope. The energyfilter removes the inelastically scattered electrons which increases the accuracy of the measurements and makes possible to study thicker specimen areas. The CBED patterns were recorded at different temperatures to study the effect of the differences in thermal expansion coefficients. The arrangement of the higher order
Laue zone (HOLZ) lines in the central disc was simulated using a fully dynamical Bloch wave program. A refinement algorithm was used to vary the strain state in the simulation until a best fit between the experimental and simulated patterns was obtained. The results indicate that in the thin films strains of up to $0.5 \%$ can occur. Strong deviations from a simpie equibiaxial strain state were observed in a polycrystalline $\langle 111\rangle$ textured film. A sensitivity to variations in lattice parameter of approximately 10-4 was obtained. Possible stress relaxations in the thin TEM sections will be discussed.

MS10.03.06 MEASUREMENTS OF SPATIAL DISTRIBUTION OF STRAIN IN QUANTUM WIRE STRUCTURES BY COHERENT GRATING X-RAY DIFFRACTION. Qun Shen, Cornell High Energy Synchrotron Source (CHESS), Cornell University, Ithaca, New York

One of the fundamental elements for better understanding and better engineering of nanostructure materials, such as quantum wires and dots, is the knowledge of intrinsic crystalline quality and the state of strain in these ultra small structures. In many cases, the effect of crystal lattice distortion on electronic band structures can be equally or more important compared to quantum confinement effects.

In recent years there are increasing interests in using highresolution $x$-ray diffraction to study the structures of these quantum wire and dot arrays. Constructive interference among the wires or the dots within the coherence width of an $\mathrm{x}$-ray beam produces diffraction satellite peaks around each crystal Bragg reflection. This phenomenon of Coherent Grating X-ray Diffraction (CGXD) enhances the scattering signal from individual wires or dots, much like the case of large-unit-cell crystallography. The grating $x$-ray diffraction pattern contains the information not only on geometric surface profiles of the wires or dots, but also on possible imperfections in the array, and crystalline registry with respect to substrate. In addition, the coherent interference within the individual wire or dot can give rise to distinct satellite peak intensity modulations in reciprocal space that are determined by the spatial distribution of the interfacial strain field. Thus this method can provide a way of directly mapping the strain distribution in a quantum wire. It also eliminates or greatly reduces the usual problem of poor strain sensitivity due to the inherent size-broadening in diffraction from small crystals.

We have applied the CGXD technique to various nanostructure arrays, including a series of 10 nm-thick InGaAs/ $\mathrm{GaAs}(001)$ quantum wire samples, with wire widths ranging from 50 to $300 \mathrm{~nm}$ and array period from 400 to $1000 \mathrm{~mm}$. Our measured values of strain in these wires show a substantial increase when the wire width becomes smaller. This result strongly suggests that the strain is the principle cause for the extra blue shifts in the photoluminescence spectra of the quantum wires. 